\title{
Re-implantation of suboptimal prostate seed implantation: technique with intraoperative treatment planning
}

\author{
Laura Doyle, MS', Adam J. Hesney, BBA', Katherine L. Chapman, MS', Haisong Liu, PhD', Perry R. Weiner, DO², \\ Adam P. Dicker, MD, PhD', Yan Yu PhD, MBA! Timothy N. Showalter, MD³ \\ IDepartment of Radiation Oncology, Jefferson Medical College of Thomas Jefferson University, Kimmel Cancer Center, Philadelphia, \\ 2Department of Urology, Jefferson Medical College of Thomas Jefferson University, Kimmel Cancer Center, Philadelphia, ${ }^{3}$ Department \\ of Radiation Oncology, University of Virginia, Charlottesville, VA, USA
}

\begin{abstract}
Purpose: Post-implant dosimetry following prostate seed implantation (PSI) occasionally reveals suboptimal dosimetric coverage of the gland. Published reports of re-implantation techniques have focused on earlier-generation techniques, including preplanned approaches and stranded seeds. The purpose of this case report is to describe a customizable approach to perform corrective re-implantation using loose seeds and intraoperative planning technique.

Material and methods: This case report describes a 63-year-old male with favorable risk prostate adenocarcinoma receiving PSI. Thirty day post-implant dosimetric evaluation revealed suboptimal coverage of the base of the gland. Using guidance from post-implant CT-images and real-time planning, the patient received a corrective re-implantation with intraoperative planning.

Results: Post-implant dosimetry after re-implantation procedure with intraoperative planning yielded improved target volume coverage that achieved standard dosimetric criteria.

Conclusions: Re-implantation as a salvage treatment technique after sub-optimal PSI is a valid treatment option performed with intraoperative real-time planning.

Key words: low-dose-rate brachytherapy, prostate cancer, re-implantation, salvage therapy, seeds.

\section{Purpose}

Standard treatment guidelines for patients with low-risk prostate cancer include active surveillance, radical prostatectomy, external beam radiation therapy and brachytherapy (seeds, High-Dose-Rate: HDR) [1]. Over the past several years, transrectal ultrasound guided (TRUS) brachytherapy with permanent implantation of seeds (PSI) has proven to be an effective treatment, especially for those patients with low risk cancers [2,3]. Freedom from biochemical failure is the criterion most commonly used to measure the effectiveness of a given implantation procedure, with failure defined as a rise in PSA of $2 \mathrm{ng} / \mathrm{mL}$ above the post-treatment nadir [4]. As PSI techniques have developed further, it has become increasingly apparent that treatment success is closely associated with the dosimetric quality of the implant [5-7]. A post-implant CT scan allows for evaluation of implant quality using measures such as the D90 (highest dose in Gy that covers $90 \%$ of the prostate target volume) and the V100 (percentage of prostate target volume encompassed by $100 \%$ of the prescription dose). The dose to surrounding organs, most notably the urethra and rectum, is also measured.

Post-implant dosimetry sometimes reveals that a portion of the prostate has been under-dosed. Without proper dose coverage, the patient may be more likely to experience a recurrence [8] and thus further treatment options should be explored. In this scenario, the patient may choose to undergo a re-implantation procedure whereby the under-dosed region of the gland may be targeted with corrective addition of seeds. However, repeat PSI poses its own set of difficulties primarily due to the presence of active seeds placed in the initial procedure; these seeds cannot be well-visualized on ultrasound [9] and the planning can be complex and error-prone. While the previously implanted seeds are visible on a $\mathrm{CT}$ scan, the $\mathrm{CT}$ is not directly compatible with the intraoperative planning software $[10,11]$. As a result, the two imaging techniques must be implemented together, so that the treatment plan for the second implant properly accounts for the dose already being received by the patient. 
Prior reports of corrective re-implantation techniques focused on earlier generation techniques, including preplanned approaches and stranded seeds [10-13]. Although intraoperative planning is now available and widely used [14], there are no published reports that describe corrective re-implantation using intraoperative planning. In the current case report, we describe an approach to perform corrective re-implantation using loose seeds and intraoperative planning.

\section{Material and methods}

The patient in this case report is a 63-year-old male with favorable risk prostate adenocarcinoma. His tumor was a clinical Stage I, T1cN0M0, prostate cancer with an elevated PSA of $2.1 \mathrm{ng} / \mathrm{mL}$ and Gleason score $6(3+3)$ in 2/12 cores. The patient had an American Urological Association (AUA) symptom index (SI) score of 1 and little to no erectile function prior to treatment. After being offered all treatment options in a multidisciplinary clinic, the patient decided to undergo PSI.

Approximately 1 month prior to prostate seed implantation, the patient was imaged via pelvic CT to estimate the volume of the prostate. The images were transferred to the Variseed ${ }^{\circledR}$ (Varian Medical Systems, Palo Alto, CA) planning system. The prostate was delineated by the radiation oncologist and the total volume $(53.4 \mathrm{~mL})$ was used to estimate the number of radioactive I- 125 seeds for the implant.

The patient was brought to the operating room (OR) for seed implantation under general anesthesia in July 2010. A Foley catheter was inserted to identify the urethra for treatment planning purposes. The patient was positioned in the dorsal lithotomy position, the perineum was cleansed and then a TRUS probe was inserted into the rectum and positioned to visualize the entire prostate. Transverse images were acquired covering the entire extent of the prostate. The radiation oncologist contoured the prostate to be used as a target volume and organs at risk (bladder and rectum). A treatment plan was generated using the Variseed $^{\circledR}$ software with a goal of delivering a minimum of $145 \mathrm{~Gy}$ to the periphery of the prostate gland. The estimated D90 was approximately 180 Gy. Treatment guidelines include rectal 1cc doses less than prescription dose and maximum ure-

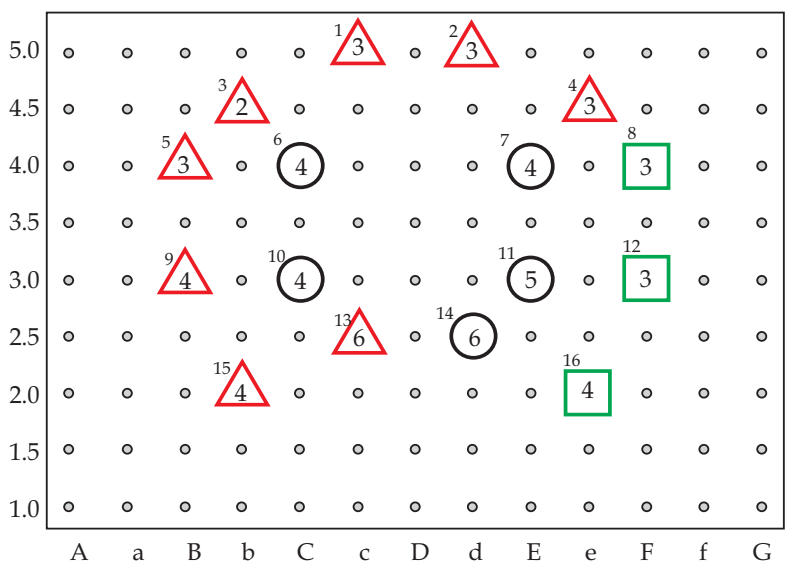

Fig. 1. Needle and seed locations on BK-Standard template for initial implant thral doses less than $150 \%$ of the prescription dose. The size of the prostate gland in the OR was approximately $39 \mathrm{~mL}$. Sixteen needles and 61 seeds were used to deliver the desired dose distribution (Figs. 1 and 2). Seed activity of $0.481 \mathrm{mCi}$ was used for the implant. A modified peripheral loading technique was used to deliver adequate dose to the prostate while minimizing dose to the bladder and urethra. Needles were placed using ultrasound guidance and ultrasound and fluoroscopic imaging were used during seed deposition. A marker cable was used to assist with defining the prostate base on fluoroscopy during the PSI procedure. The depth of the marker cable was verified of the base slice as identified on ultrasound. Both ultrasound and fluoroscopy were used throughout the procedure to verify position relative to the base of the prostate to aid in seed deposition.

The patient returned for a post implant imaging study approximately 30 days after PSI, allowing for edema of the gland to subside [15]. At this point, the patient reported an AUA SI score of 2, with quality of life score of 2. The patient was imaged via CT and images were sent to the treatment planning system for contour delineation and seed identification. The prostate and rectum were contoured. Since a Foley catheter was not inserted and the urethra is not easily identified on CT imaging, a volume called "central prostate" was contoured as a surrogate for estimating urethral doses. Post-implant dosimetry revealed unacceptably low dose coverage (V100, 71.01\%; D90, 92.9 Gy). Review of the images demonstrated that the base was underdosed and comparison to the intraoperative images showed that the base was underestimated on the intraoperative images (Figs. 3 and 4).

The option of supplementing dose through a second implant was discussed with the patient and the patient was amenable to this plan. The post implant CT images were reimported into the planning system and used as an image

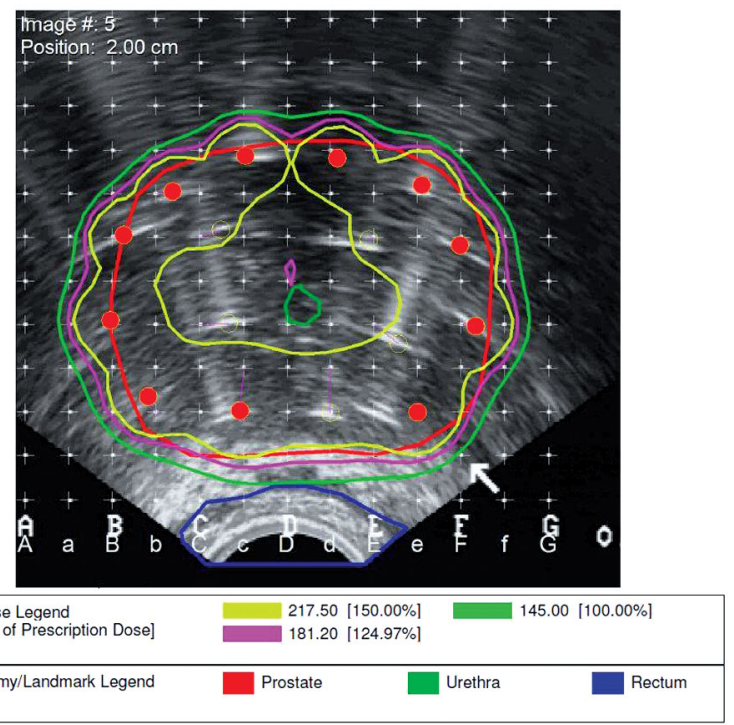

Fig. 2. TRUS image of dosimetry from intra-operative treatment plan. Contours include prostate, urethra and rectum. Isodose lines show coverage of $100 \%, 125 \%$ and $150 \%$ of 145 Gy prescription dose 


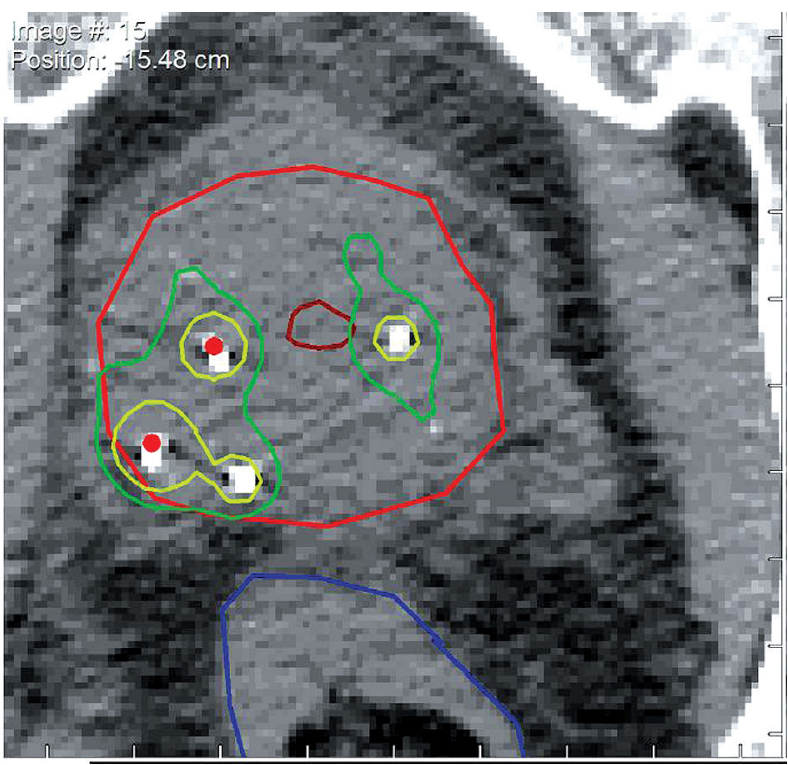

Fig. 3. Shows inferior coverage at the base of the prostate gland following PSI

set for the purpose of pre-planning. Despite the limitation that CT images have no fixed coordinate system relative to needle positions used in US imaging and that the slice thickness may vary in magnitude from US slice spacing, the approximate seed locations and quantity of additional seeds necessary to achieve the desired dose distribution were approximated using the CT image set (Fig. 5). Based on preliminary planning studies performed in the Variseed $^{\circledR}$ planning system, it was determined that approximately 20 additional seeds would be necessary to compensate for the initially under-dosed region of the gland.

\section{Results}

A hypothetical pre-plan, based on the post-implant CT, was printed and brought to the OR as a guide for the re-implantation procedure (Fig. 6). The corrective re-implantation procedure was performed approximately two months after the date of the initial implant. In the OR, the patient was set-up in the dorsal lithotomy position as described above. TRUS imaging was used to identify the gland and capture transverse images to the planning system. The prostate, urethra and rectum were again delineated. Before a new treatment plan was generated, the previously deposited seeds were loaded as a template from the initial plan and marked as active seeds. Using the printed pre-plan generated from the post-implant CT as a guide and considering the initial seed locations, a composite plan was generated requiring 20 additional seeds deposited through a total of 12 needles. For plan evaluation, decay of the previously deposited seeds was not taken into consideration and the activity per seed on the day of each respective implant, $0.481 \mathrm{mCi}$, was assigned to all seeds (Fig. 7). The quality of the implant as judged by dosimetric indicators significantly improved after re-implantation judged by CT post implant dosimetry following the second implant. The V100

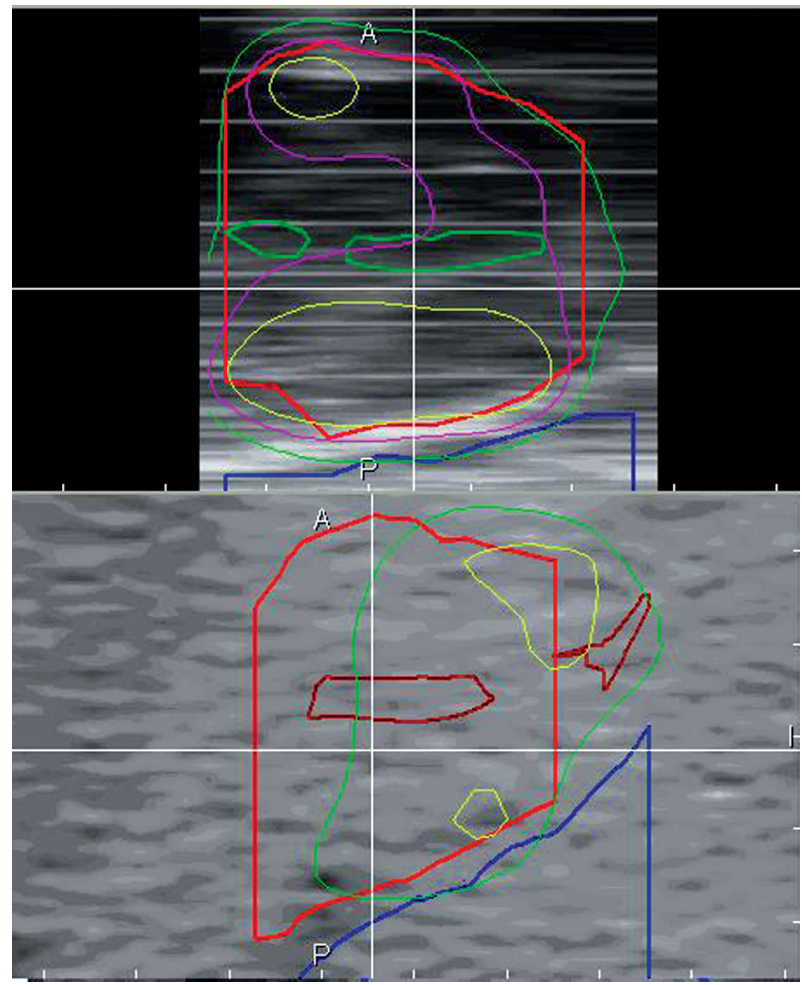

Fig. 4. Mid-sagittal views of intended coveraged indicated by TRUS (upper) and achieved coverage as indicated by 30-day post-operative CT (lower)

improved from $71.01 \%$ to $96.88 \%$ and the D90 increased from 92.9 Gy to $173.24 \mathrm{~Gy}$. These results were close to the planned D90 and V100 values of 182.23 Gy and $99.46 \%$, respectively. After re-implantation, the dose received by the urethra and rectum remained within the acceptable range. Final post-implant dosimetry was performed 30 days after the corrective re-implantation (Fig. 8).

\section{Discussion}

Although relatively uncommon in high-volume programs, prostate seed implant (PSI) procedures may result in sub-optimal dosage identified on post-implant dosimetry, requiring further treatment decisions to be made. The options for a suboptimal PSI with lower than adequate doses include a wait-and-see approach, supplemental external beam radiation therapy to the entire prostate target volume or a corrective re-implantation procedure to an underdosed region(s) of the prostate. Each approach carries its own risks, including deleterious treatment-related side effects and possible disease recurrence [16]. After being fully informed of his options, the patient in this case decided to correct the under-dosed region of the prostate through a second PSI procedure that incorporated intraoperative planning techniques. As demonstrated by this case, re-implantation may be a preferred course of action in some cases where a specific region of the prostate can be identified to place additional seeds. In this case, the base was underestimated in the original PSI, so the corrective re-implantation delivered supplemental seeds to the base. 

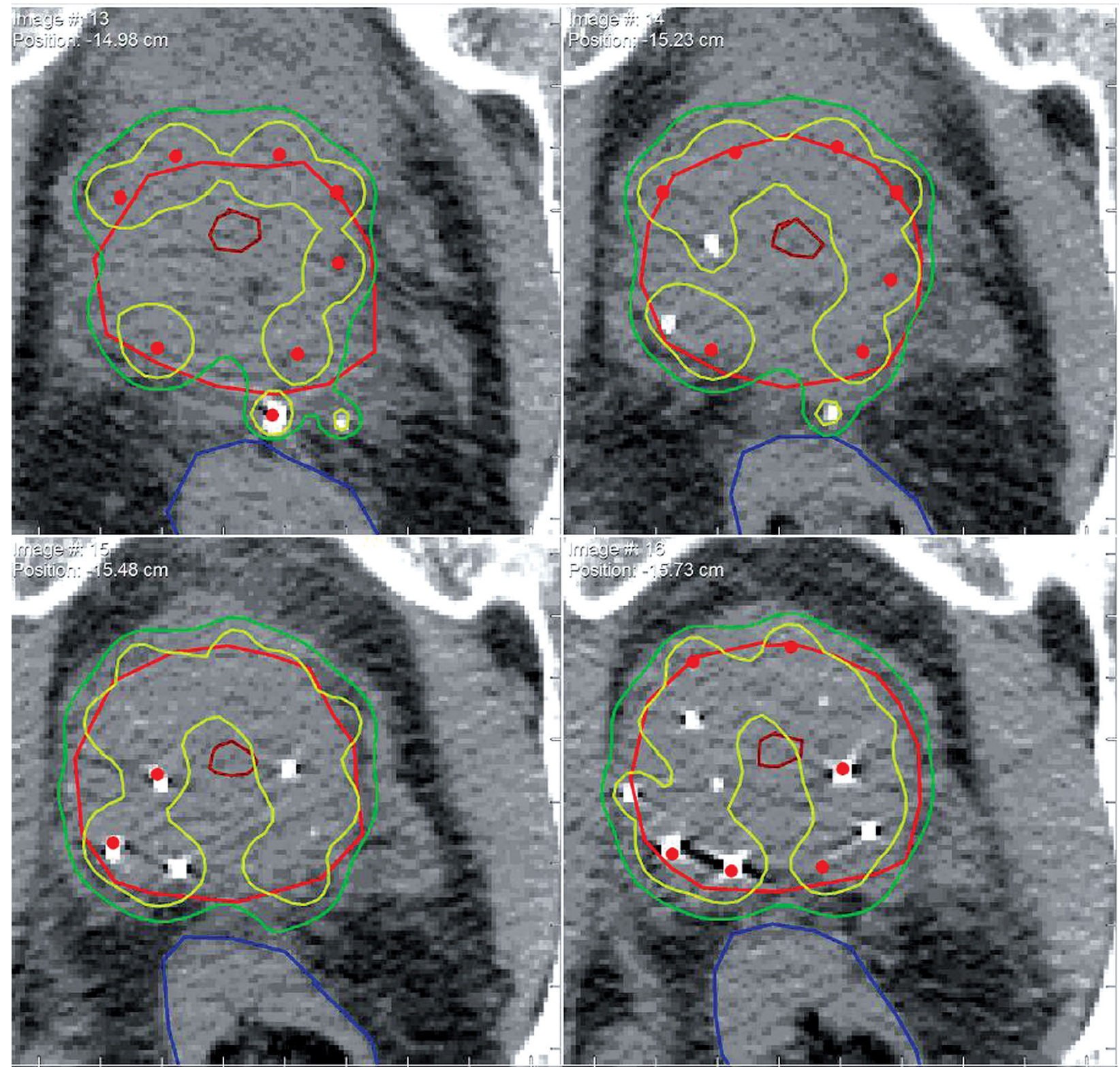

Fig. 5. Shows use of CT images to calculate approximate location and number of seeds needed for supplemental implant. Approximate positions on pre-implant plan served as a guide for seed locations during intra-operative planning

As previously stated, re-implantation presents obstacles that do not arise in a typical PSI procedure. Due to limitations inherent to the treatment planning technology, it may be difficult to ascertain the precise location of existing seeds, thereby making it more challenging to devise an adequate treatment plan. The approach used here, which incorporated both the post-implant CT obtained after the original PSI as well as intraoperative real-time planning proved to be effective in improving the dosimetric quality of the implant and ultimately the clinical outcome for the patient. This approach is flexible, customizable and can be performed using standard tools available for intraoperatively-planned brachytherapy with TRUS images.

Prior studies have described re-implantation primarily using a CT guided pre-planning approach $[10,12]$, but previous reports did not describe techniques that incorporate intra- operative planning. For these procedures, a post-implant CT scan was the primary tool used in creating a treatment plan, allowing for existing seeds to be visualized and new seeds to be planned for re-implantation. However, the method does not afford the same flexibility as the intraoperative method used in this case. Because the treatment is planned approximately one month before the actual procedure, the pre-planning approach has some important limitations. The main challenge is placing the patient in the exact same position used during planning; it is often difficult to replicate this position precisely. Also, there may be changes in prostate volume between planning and procedure, leading to deviations from intended seed location and dosimetry. Intraoperative planning allows for more flexibility such that needle locations and seed placement can be adjusted as needed during the procedure and dose distributions may be updated continuously 


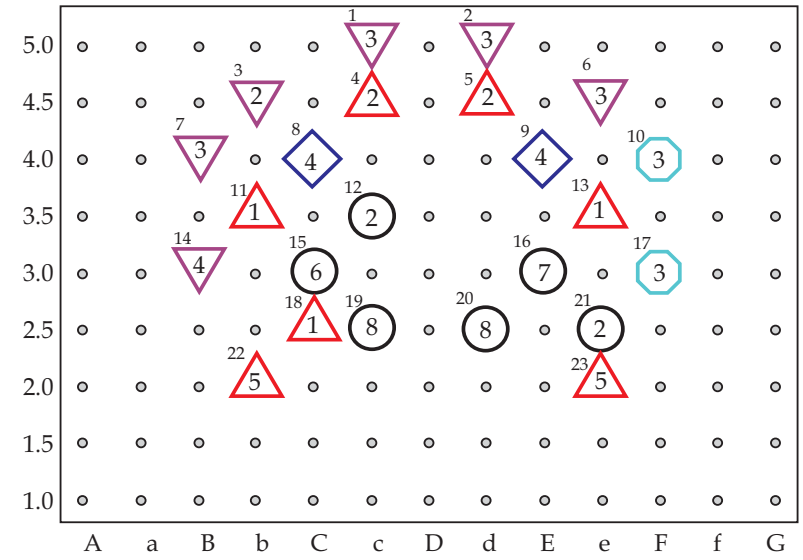

Fig. 6. Planned needle and seed locations for second implant considering seed locations from first implant

during the corrective PSI [17-19]. Furthermore, previous studies have shown that intraoperative planning leads to a closer match between OR and post-implant dosimetry [20]. This paper thus describes re-implantation utilizing a contemporary technique with real-time planning.

It should be noted that supplemental EBRT is another option for re-implantation after an unsatisfactory PSI [21]. In cases where the prostate target volume is routinely underdosed, supplemental EBRT may be preferable to corrective re-implantation. In cases where the patient reports severe urinary morbidity after the first PSI procedure or when the maximum dose delivered to the urethra approaches or

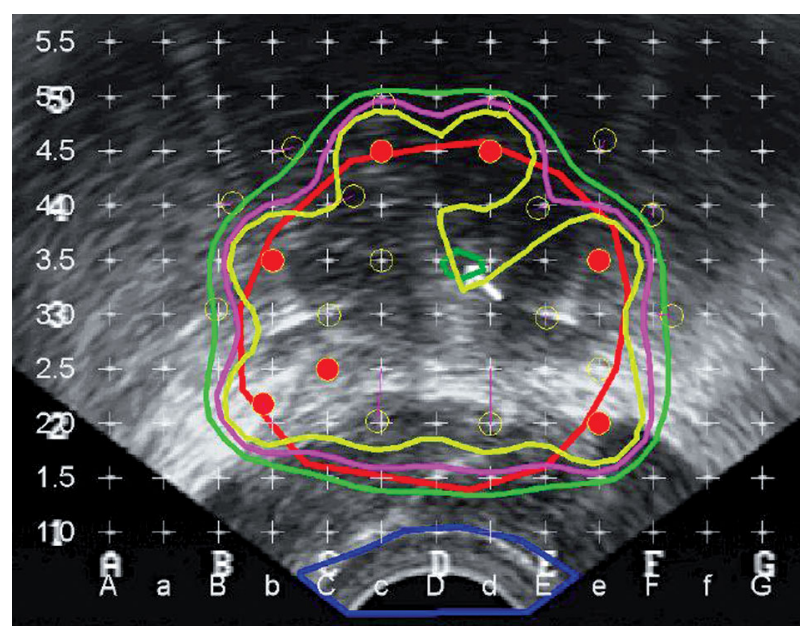

Fig. 7. Intra-operative plan considering seeds from initial implant

exceeds the tolerance level, it may be prudent to avoid reimplantation. In a situation such as the case presented in this study, where the area of underdosage is limited to a defined region, we recommend that corrective re-implantation using an intraoperative, real-time planning approach be employed to achieve target cumulative doses.

\section{Patient Follow-up}

The patient feels well 1.5 years after the procedure and is no longer on alpha blocker therapy. His follow-up PSA testing shows that it is decreased to $0.70 \mathrm{ng} / \mathrm{mL}$, from baseline value of $2.0 \mathrm{ng} / \mathrm{mL}$.
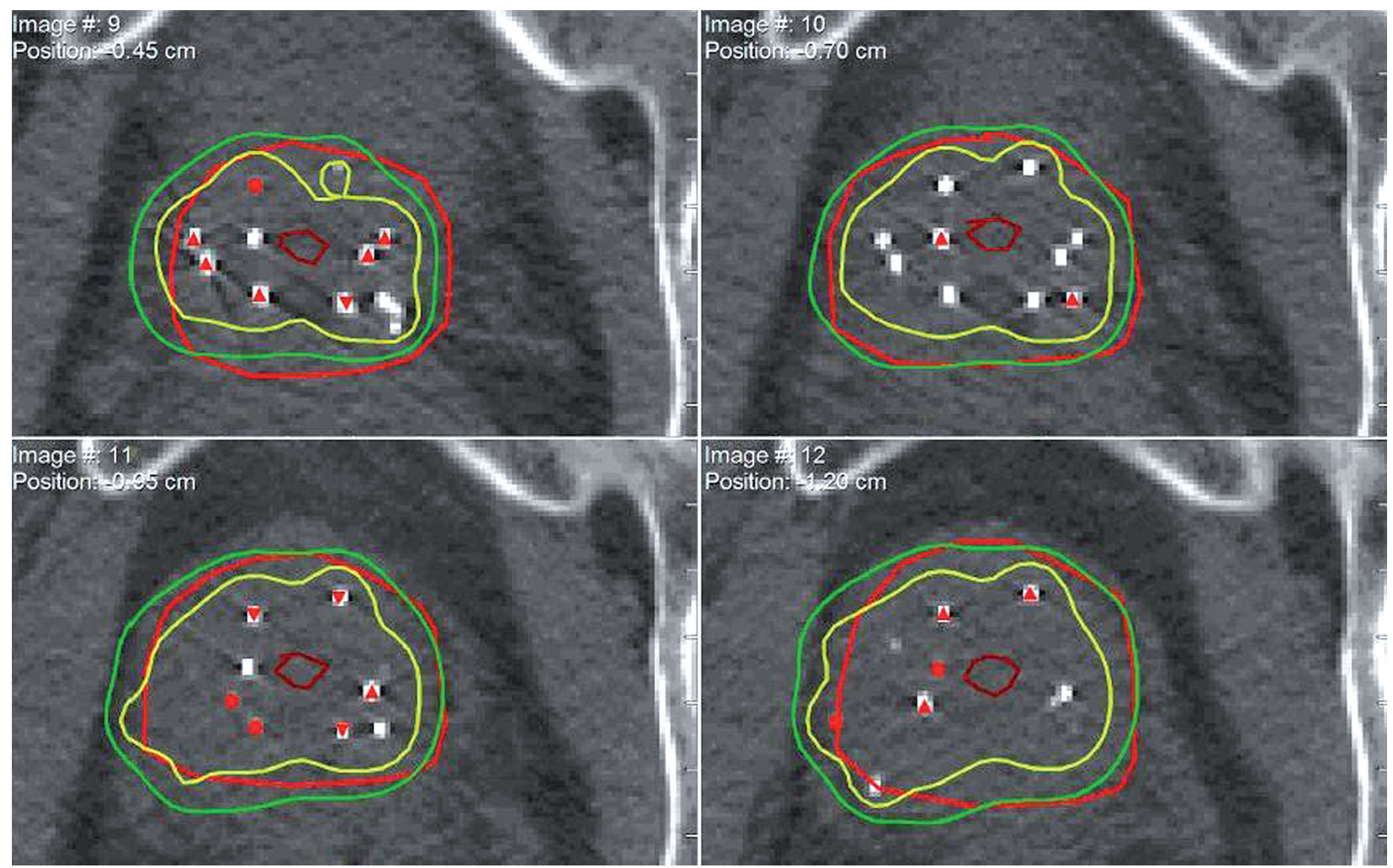

Fig. 8. Post implant dosimetry from both initial and salvage PSI 


\section{Conclusions}

Re-implantation as a salvage treatment technique after sub-optimal prostate seed implantation is a valid treatment option and is made easier by an intra-operative real time planning technique. The method described in this paper of using the post implant $\mathrm{CT}$ as a pre-implant planning tool to estimate seed number and locations and intra-operative planning for real time feedback of current and planned seed locations is feasible. This technique may be adapted for other cases of sub-optimal dosimetry following PSI. Much caution must be placed on doses to the urethra and rectum following the initial and subsequent implants. Intra-operative planning allows the user more flexibility in patient positioning, locating previously deposited seeds and real time dosimetry of supplemental seed deposition.

\section{References}

1. Scherr D, Swindle P, Scardino P. National Comprehensive Cancer Network guidelines for the management of prostate cancer. Urology 2003; 61: 14-24.

2. Kollmeier MA, Stock RG, Stone N. Biochemical outcomes after prostate brachytherapy with 5-year minimal follow-up: Importance of patient selection and implant quality. Int J Radiat Oncol Biol Phys 2003; 57: 645-653.

3. Hinnen KA, Battermann JJ, van Roermund JG et al. Long-term biochemical and survival outcome of 921 patients treated with I-125 permanent prostate brachytherapy. Int J Radiat Oncol Biol Phys 2010; 76: 1433-1438.

4. Henry AM, Al-Qaisieh B, Gould K et al. Outcomes following iodine-125 monotherapy for localized prostate cancer: The results of leeds 10-year single-center brachytherapy experience. Int J Radiat Oncol Biol Phys 2010; 76: 50-56.

5. Ash D, Al-Qaisieh B, Bottomley D et al. The correlation between D90 and outcome for I-125 seed implant monotherapy for localised prostate cancer. Radiother Oncol 2006; 79: 185-189.

6. Potters L, Huang D, Calugaru E et al. Importance of implant dosimetry for patients undergoing prostate brachytherapy. Urology 2003; 62: 1073-1077.

7. Stock RG, Stone NN. Importance of post-implant dosimetry in permanent prostate brachytherapy. Eur Urol 2002; 41: 434-439.

8. Nag S, Beyer D, Friedland J et al. American brachytherapy society (ABS) recommendations for transperineal permanent brachytherapy of prostate cancer. Int J Radiat Oncol Biol Phys 1999; 44: 789-799.

9. Han BH, Wallner K, Merrick G et al. Prostate brachytherapy seed identification on post-implant TRUS images. Med Phys 2003; 30: 898-900.

10. Hughes L, Waterman FM, Dicker AP. Salvage of suboptimal prostate seed implantation: Reimplantation of underdosed region of prostate base. Brachytherapy 2005; 4: 163-170.

11. Keyes M, Pickles T, Agranovich A et al. 125I reimplantation in patients with poor initial dosimetry after prostate brachytherapy. Int J Radiat Oncol Biol Phys 2004; 60: 40-50.

12. Koutrouvelis $\mathrm{P}$, Hendricks F, Lailas $\mathrm{N}$ et al. Salvage reimplantation in patient with local recurrent prostate carcinoma after brachytherapy with three dimensional computed tomographyguided permanent pararectal implant. Technol Cancer Res Treat 2003; 2: 339-344.

13. Bice WS Jr, Freeman JE, Russell LF Jr et al. Use of image coregistration in salvage prostate brachytherapy. Tech Urol 2000; 6 : 151-6.

14. Raben A, Sammons S, Sim S et al. Initial comparison of inverse optimization, modified peripheral technique, and geometric optimization as real-time intraoperative computer planning options for permanent seed implantation of the prostate. Brachytherapy 2007; 6: 238-245.

15. Waterman FM, Dicker A. Impact of postimplant edema on V100 and D90 in prostate brachytherapy: Can implant quality be predicted on Day 0? Int J Radiat Oncol Biol Phys 2002; 53: 610-621.

16. Brachman DG, Thomas T, Hilbe J et al. Failure-free survival following brachytherapy alone or external beam irradiation alone for T1-2 prostate tumors in 2222 patients: Results from a single practice. Int J Radiat Oncol Biol Phys 2000; 48: 111-117.

17. Matzkin H, Kaver I, Bramante-Schreiber L et al. Comparison between two iodine-125 brachytherapy implant techniques: Pre-planning and intra-operative by various dosimetry quality indicators. Radiother Oncol 2003; 68: 289-294.

18. Matzkin H, Kaver I, Stenger A et al. Iodine-125 brachytherapy for localized prostate cancer and urinary morbidity: A prospective comparison of two seed implant methods-preplanning and intraoperative planning. Urology 2003; 62: 497-502.

19. Stock RG, Stone NN, Lo YC. Intraoperative dosimetric representation of the real-time ultrasound-guided prostate implant. Tech Urol 2000; 6: 95-98.

20. Stone NN, Hong S, Lo YC et al. Comparison of intraoperative dosimetric implant representation with postimplant dosimetry in patients receiving prostate brachytherapy. Brachytherapy 2003; 2: 17-25.

21. Sahgal A, Roach M. Permanent prostate seed brachytherapy: a current perspective on the evolution of the technique and its application. Nat Clin Pract Urol 2007; 4: 657-670. 